\title{
A 1.2V Wide-Band Reconfigurable Mixer for Wireless Application in 65nm CMOS Technology
}

\author{
Nisha Gupta, A R Aravinth Kumar, Ashudeb Dutta, Shiv Govind Singh \\ Department of Electrical Engineering, IIT Hyderabad, Aandhra Pradesh, India \\ Email: 31.nisha@gmail.com, akarmephd@yahoo.com, (asudebdutta, sgsingh)@iith.ac.in
}

\begin{abstract}
This paper presents a wideband (WB) reconfigurable down-conversion mixer for multi-standard wireless receivers. The proposed mixer is re-configurable between active mixer and passive mixer modes. Reconfigurability is made through switching the input signal between gate and source terminal of input transistors and enabling/disabling the transimpedance stage at the output. The CMOS transmission gate (TG) switches are designed to provide optimum headroom in this low voltage design. The proposed circuit is designed in UMC $65 \mathrm{~nm}$ RFCMOS technology with $1.2 \mathrm{~V}$ supply voltage. From the simulation results, the proposed circuit shows conversion gain of $29.2 \mathrm{~dB}$ and $25.5 \mathrm{~dB}$, noise figure of $7.6 \mathrm{~dB}$ and $10.2 \mathrm{~dB}, I I P_{3}$ of $-11.9 \mathrm{dBm}$ and $6.5 \mathrm{dBm}$ in active and passive mode respectively. Hence this circuit will be much helpful in multi-standard receiver design in IoT perspective.
\end{abstract}

keywords- Reconfigurable; passive; active; mixer; multistandard; receivers;

\section{INTRODUCTION}

Emerging Internet of Things (IoT) enabled platform demands multi-mode multi-standard transceivers to enhance the performance through seamless connectivity between zigbee, bluetooth, Wi-Fi, UWB and cognitive radio interface. The easiest solution is to put multiple separate radio to above need[1]-[6], however, in real scenario only one of the mode function at a time. So above approach is power hungry, costly and take more area. Therefore, to get a cost effective solution, a re-configurable single radio (that can configure to multi-mode as need basis) would be the best choice. To make the radio reconfigurable researchers introduce the RF transceiver front-end with reconfigurable LNA[7]-[8], PA, PLL[9], mixer[10]-[11], and filter[12] etc. Among them, our emphasis is to design a Mixer that can provide reconfigurlibity on the performances like gain, linearity, noise figure and bandwidth selection. Most of proposed reconfigurable mixer have shown gain variability and Bandwidth tuning[13]-[14] through current variation, load tuning etc[10]-[12]. In such designs, multi channel sensing and signal strength adaptability are the main target and are designed for single standard operation. However, in multi-mode IoT systems, the other performances like noise figure, linearity reconfiguration are need to be incorporated in mixer design.In this regard, we propose a re-configurable down conversion mixer which is switchable between active and passive mode depends on the performance requirement as shown in Fig. 1.

The prime features of the mixer are:

1) Reconfiguration in single circuitry between active and passive modes with gain and noise tunability.

2) Common source topology is used for active mixer with a current source at the bottom. Transmission

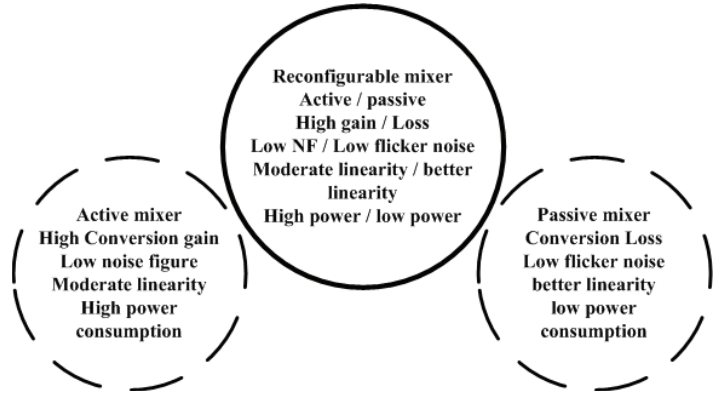

Fig. 1. Trade-offs between active and passive mixers

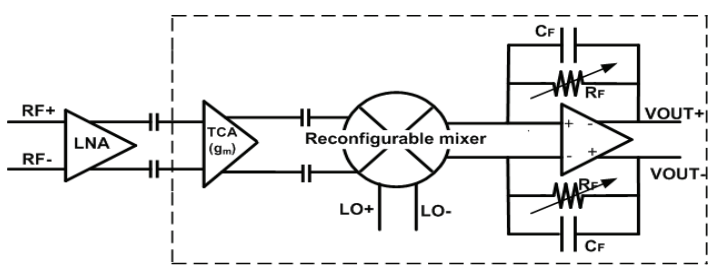

Fig. 2. Wide-band receiver front end.

gate is used as a resistance to provide optimum headroom. The current commutating passive mixer is designed by using four switching(LO) MOS with resistive degeneration.

3) PMOS switch is used to switch on or off Transimpedance amplifier.

Upto the best of our knowledge, first time one such work is being reported in IoT perspective and detail operation are described in following paragraphs as section II details the proposed architecture and simulation results are explained in Section III.

\section{ARCHITECTURE OVERVIEW}

The block diagram of wide-band RF front-end (demodulator) is shown in Fig. 2. The first building block in the wide band RF front-end is the LNA. So it is required to design high input impedance $g_{m}$ stage to avoid loading effect. Simultaneously $g_{m}$ stage provides high isolation between IF, LO and RF. The differential ended RF input is taken by RF balun using $50 \mathrm{ohm}$ input impedance termination. All the signal paths are fully differential in style to suppress the common mode noise and second order harmonic.

This block consists of two types of mixer in single circuitry. Reconfigurable (Active/Passive) mixer consists of RF transconductance amplifier, switching stage and load stage. 


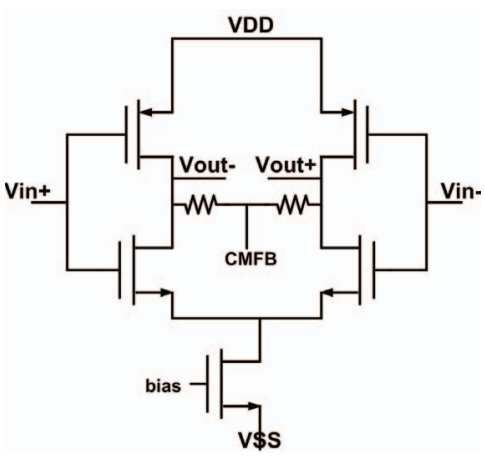

Fig. 3. Transconductance amplifier.

Tranconductance amplifier that converts the RF input voltage into current is DC decoupled to switching stage. Mixer core output is coupled to transimpedance stage. IF voltage is built at the output of transimpedance amplifier after the RF current gets commutated in switching stage and passes to first order RC low pass filtering. TIA offers a virtual node for the $g_{m}$ stage, resulting in high linearity. TIA power will be switch on or off in passive and active case respectively to save the power consumption. Unlike narrow-band designs, $C_{P A R}$ (parasitic capacitance at the output node of the transconductance stage ) is minimized to allow a less stringent noise specification upon the op-amp which favourably gets translated into lower power consumption circuit design.

\section{A. Transconductance Amplifier}

Fully differential CMOS transconductor is employed to convert the input RF voltage signal to RF current as shown in Fig. 3. Post which the current signal is fed to the switching stage ensuring that second order nonlinearity is reduced by using fully differential topology. TCA is modeled with a frequency dependent source impedance $Z_{S}(\omega)$ is desired to minimize the signal loss and enhance its linearity performance according to (1),(2) [5]

$$
\begin{gathered}
I I P_{2} \approx K_{a} \frac{Z_{L}\left(\omega_{1}\right) Z_{s}\left(\omega_{1}-\omega_{2}\right)}{Z_{L}\left(\omega_{1}-\omega_{2}\right) f\left[Z_{L}\left(\omega_{L O}-\omega_{1}\right)\right]} \\
I I P_{3} \approx K_{b} \frac{Z_{L}\left(\omega_{L O}-\omega_{1}\right) Z_{s}\left(2 \omega_{1}-\omega_{2}\right)}{Z_{L}\left[\omega_{L O}-\left(2 \omega_{1}-\omega_{2}\right)\right] g\left[Z_{L}\left(\omega_{L O}-\omega_{1}\right)\right]}
\end{gathered}
$$

Where TIA stage is modeled as the load impedance of mixer $Z_{L}(\omega), \omega_{1} \omega_{2}$ represent the two nearby RF frequencies of input tones, and $\omega_{L O}$ represents the LO frequency.

The common mode voltage is designed at VDD/2 for getting maximum swing. By setting this common mode voltage, current can be minimized and parasitic capacitance at the output nodes of transconductor is optimized to the smallest possible value thereby increasing the output impedance, for the purpose of improving the NF. Transconductance stage gain also reduces the overall noise of front end receiver. Common mode feedback for the tranconductance stage needs to be carefully designed for proper operating point.

\section{B. Common Source Reconfigurable Mixer}

In this embodiment, modulator circuit is reconfigured between active and passive modes by switching between the

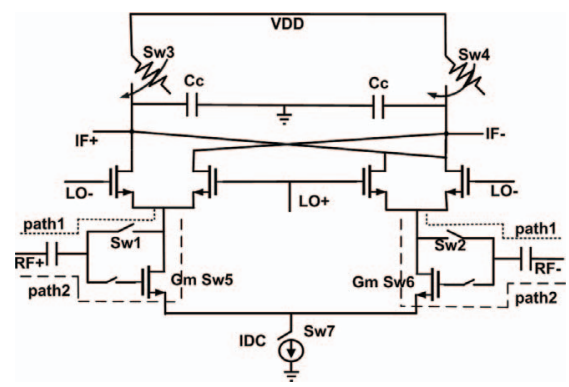

Fig. 4. Reconfigurable mixer.

output load, DC power supply and Gm stage (Gm MOS of active case) and current source shown in Fig. 4. Common source configuration is chosen because of compatibility with cases.

In passive mode, the frequency mixer or modulator circuit is simply composed of four NMOS transistors characterized by resistance (Ron) when switched on. Accordingly, in order to make the common-source input stage configuration suitable for both active and passive mode topologies, switch (Sw1-2) are implemented using PMOS which have been added between the gate and drain of the common source transistor (Gm MOS)as shown in Fig. 4. TCA differential output current is applied at the drain of the transistor Mp1 and Mp2 (PMOS switch 1-2) to route to the mixer core for mixing in the current domain. Vlogic high or low is given to Mp1 and Mp2 to configure reconfigurable mixer to operate in an active/passive mode as shown in Fig. 5(a). Specifically when there is no current flowing through the mixer core, Vlogic is set to zero, thus causing input signal to flow directly through the switching stage as shown in path 1 to mix with LO signal. Width of PMOS is chosen to provide degeneration resistance, thus turning the overall mixer topology into a passive mode as shown in Fig. 6(a). Transistors thus operate as switch 1-2 as well as degeneration resistance $R_{d e g}$ (switch 1-2 resistance), thereby increasing linearity of passive mixer [6]. Capacitor Cc is a high-frequency compensation capacitor used to suppress the noise at higher frequency. The output signal is supplied from the mixer core without any load and directly coupled with transimpedance amplifier. The voltage conversion gain of passive mixer is

$$
V C G=\frac{2}{\Pi} * g_{m} * Z_{F}
$$

where $Z_{F}$ is the feedback impedance of transimpedance amplifier, $C_{F}$ parallel with $R_{F}$ and $g_{m}$ is the transconductance of transconductance amplifier.

Resistive switches 3-4 designed using transmission gate, made of PMOS and NMOS switch, are fully turned off ensuring that output current of switching quard directly goes to the transimpedance amplifier without any coupling capacitor. Switches 5-7 designed using NMOS will also be off when this circuit operates in passive mode.

In active mode, input stage of the frequency mixer or modulator circuit have a configuration that is a common source topology. This topology is chosen to provide better gain and low noise figure. Double balance gilbert cell architecture is used in active configuration when there is current flowing 


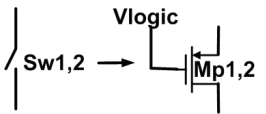

(a) PMOS switch

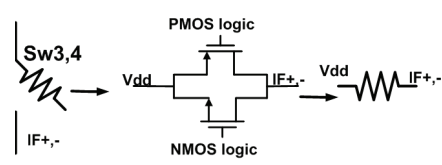

(b) Resistive switch
Fig. 5. Switch implementation using MOS

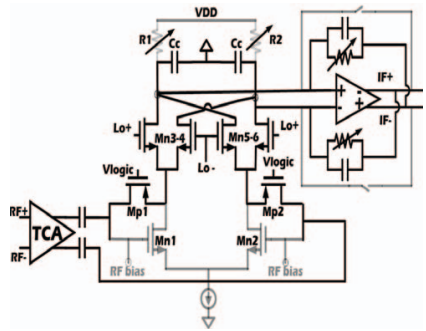

(a) Passive mode

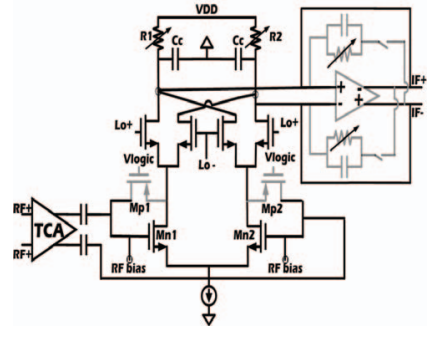

(b) Active mode
Fig. 6. Reconfigurable mixer in passive and active mode

through input $(\mathrm{Gm})$ MOS Mn1 and Mn2 (Sw 5-6). The bias voltage can be selected to control parameters of input stage or switching operation of Gm MOS switch 5-6 as shown in path 2. The Gm of MOS Mn1 and Mn2 can be changed by changing the value of bias voltage, thus varying the gain of mixer. The optimum value of bias voltage is so desired so that mixer consumes a minimal amount of current. Switch 7 has been designed using NMOS which is biased in saturation region to provide current source. Thus turning the overall topology into an active mode as shown in Fig. 6(b).

Transmission gate is used as a resistive switch connected between VDD and IF output as shown in Fig. 5(b). W/L of PMOS and NMOS is chosen so that some voltage drop occurs across it and act as a resistance. Transmission gate total resistance is $R$ tol $=R_{P} M O S \| R_{N} M O S$. As it is connected to VDD so it acts as resistive load and Capacitor $\mathrm{Cc}$ is provided to act as a low pass filter in active mode operation of reconfigurable mixer. Gain of active mixer can be tuned by changing the resistance of transmission gate. The output of active mixer is directly passed to the output stage without going to TIA.

\section{Transimpedance Amplifier}

A simplified schematic of transimpedance amplifier is shown in Fig. 7(a). TIA consists of an operational transconductance amplifier with a feedback $R_{F} C_{F} . R_{F}$ and $C_{F}$ value is set according to IF frequency. A two stage miller compensated OTA topology is chosen for TIA design as shown in Fig. 7(b). First stage to provide high gain and second stage for high swing. So that structure can obtain both, high output swing and low input referred noise. Transimpedance amplifier is used to convert current to voltage output in passive mode operation. The TIA stage serves as load and anti-aliasing filter for the passive mixer. The TIA is designed in such a way so that very low impedance is provided at the passive mixer output. TIA input impedance is given by

$$
Z_{i n}(f)=\frac{2}{A(f)} * \frac{R_{F}}{1+2 \Pi R_{F} C_{F}}
$$

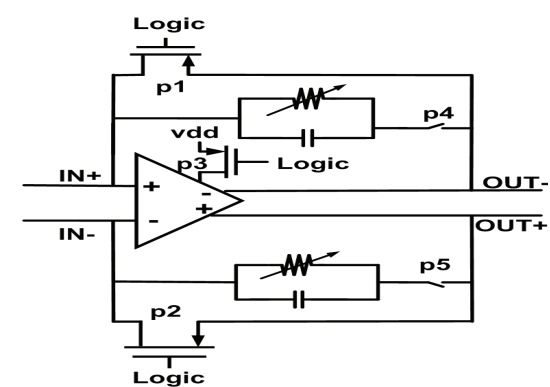

(a) Schematic of TIA stage

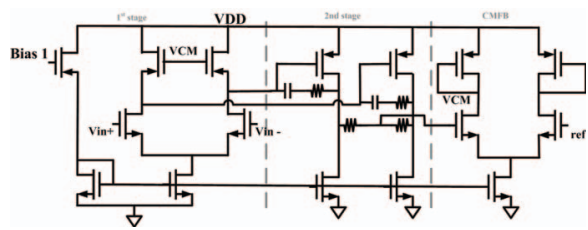

(b) Schematic of OTA

Fig. 7. Transimpedance amplifier

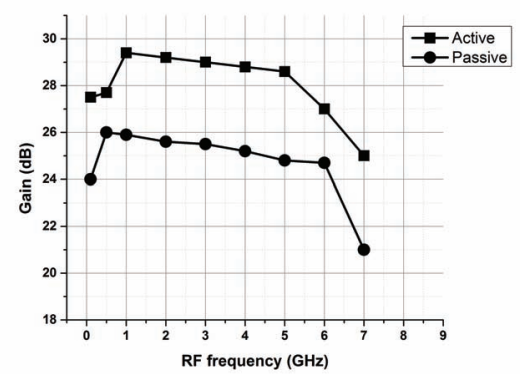

Fig. 8. Simulated conversion gain reconfigurable mixer vs RF frequency.

Where $A(f)$ is the open loop gain of the OTA. Due to high gain OTA bandwidth is limited and high frequency components suffer high impedance. In order to filter out high frequency components $C_{F}$ is inserted. This is done for all signal current to flow into feedback $R_{F} C_{F}$ from the mixer core. The TIA draws a total of $3.3 \mathrm{~mA}$ from the supply. In case of active mixer operation TIA will be switched off to save power. In case of active mixer operation TIA will be switched off by switching off p3 switch to save power and p1, p2 will be on. p4 and p5 also implemented using MOS and switch on or off in case of passive and active respectively. The gain of the TIA can be tuned by changing the value of $R_{F}$ and it provides another degree of freedom to configure the gain of the downconverter.

\section{Simulation Results}

Based on the qualitative description of the building blocks, using their insights related to operation, the RF front-end demodulator is simulated in CMOS $65 \mathrm{~nm}$ process. The voltage conversion gain plot is shown in Fig. 8 with respect to RF frequency at $5 \mathrm{MHz}$ IF. The voltage conversion gain is close to $29.2 \mathrm{~dB}$ and $25.5 \mathrm{~dB}$ for acive and passive case respectively.

The simulated double side band noise figure at $2.45 \mathrm{GHz}$ is shown in Fig. 9. In addition, the corner frequency is less than $100 \mathrm{KHz}$ in passive mode operation. Simulated noise figure for active and passive is $7.6 \mathrm{~dB}$ and $10.2 \mathrm{~dB}$ @ $5 \mathrm{MHz}$ respectively. 
TABLE I. SIMULATION RESULTS AND COMPARISON

\begin{tabular}{|c|c|c|c|c|c|c|c|c|c|c|}
\hline Parameters & $\begin{array}{l}\text { Active } \\
\text { (This work) }\end{array}$ & $\begin{array}{l}\text { Passive } \\
\text { (This work) }\end{array}$ & [2] & [3] & [5] & {$[6]$} & [4] & {$[10]$} & [11] & [12] \\
\hline Gain $(\mathrm{dB})$ & 29.2 & 25.5 & 14.5 & 13 & 21 & $22.5-25$ & 35 & $9-24$ & $1.2-17$ & $3.5-20.5$ \\
\hline Noise figure $(\mathrm{dB})$ & 7.7 & 10.2 & 6.5 & 13.7 & 10.6 & $7.7-9.5$ & 10 & NA & $\geq 11$ & $\geq 8$ \\
\hline IIP3 $(\mathrm{dBm})$ & -11.9 & 6.57 & NA & $\geq 10.8$ & 9 & $\geq 7$ & 11 & 3.5 to -12 & 8.6 & $\leq 8.5$ \\
\hline $1 \mathrm{~dB}-\mathrm{CP}(\mathrm{dBm})$ & $-24.5 @ 5 \mathrm{MHz}$ & $-14 @ 5 \mathrm{MHz}$ & -13.8 & NA & NA & -12 & $-25.8 @ .1 \mathrm{MHz}$ & -4 to -19 & -3.7 & NA \\
\hline power $(\mathrm{mW})$ & 9.36 & 9.24 & 14.4 & 8.04 & 9.9 & $10($ mixer + TIA $)$ & 20.25 & 2.4 to 18 & 5.9 & $5.6-9.6$ \\
\hline Bandwidth(GHz) & 1 to 5.5 & .5 to 5.1 & 1 to 10.5 & $900 \mathrm{M}, 1.8-2.5 \mathrm{G}$ & .7 to 2.3 & 1.55 to 2.3 & .7 to 2.5 & 2 to 10 & 1 to 12 & .7 to 2.3 \\
\hline CMOS Technology & $65 \mathrm{~nm}$ & $65 \mathrm{~nm}$ & $65 \mathrm{~nm}$ & $65 \mathrm{~nm}$ & $180 \mathrm{~nm}$ & $180 \mathrm{~nm}$ & $130 \mathrm{~nm}$ & $130 \mathrm{~nm}$ & $130 \mathrm{~nm}$ & $180 \mathrm{~nm}$ \\
\hline Power supply & $1.2 \mathrm{~V}$ & $1.2 \mathrm{~V}$ & 1.2 & 1.2 & 1.8 & 2 & 1.5 & 1.2 & 1.2 & 1.8 \\
\hline
\end{tabular}

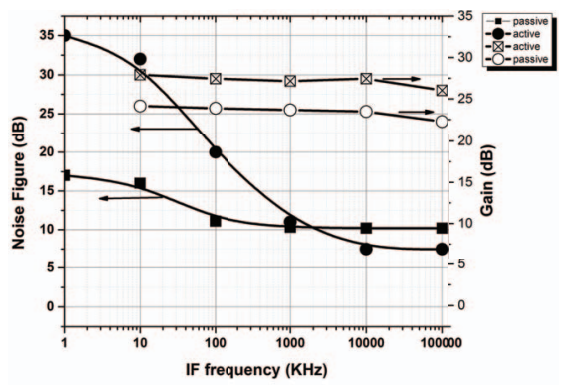

Fig. 9. Simulated noise figure and conversion gain vs IF frequency.

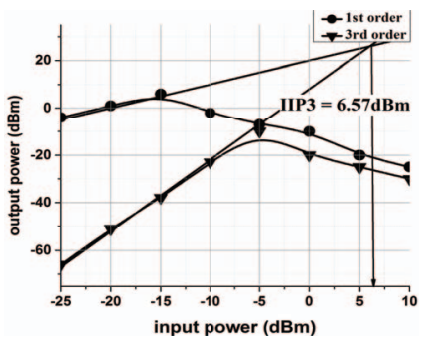

(a) $I I P_{3}$ of passive mixer.

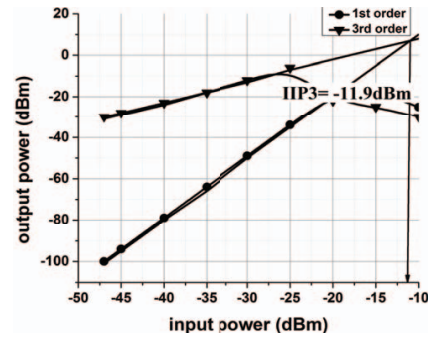

(b) $I I P_{3}$ of active mixer.
Fig. 10. Simulated linearity of reconfigurable mixer

The two tone linearity test result is shown in Fig. 10(a) for $2.4 \mathrm{GHz} \mathrm{LO}$ frequency. Due to high conversion gain at low IF, the output compression point of the OPAMP, limits the input referred linearity of the circuit. 1dB-compression point of the circuit is limited by the output swing and varies with IF frequency. The simulated $I I P_{3}$ in case of passive is $6.57 \mathrm{dBm}$ and $I I P_{3}$ of active is shown in Fig. $10(\mathrm{~b}) . I I P_{3}$ of active is $-11.9 \mathrm{dBm}$.

\section{CONCLUSiON}

In this paper, a wide band Reconfigurable mixer is simulated in $65 \mathrm{~nm}$ technology. A new concept of reconfiguration between Active / passive downconversion mixer is presented. This is an important design guideline (both topology in single circuitry) for modern baseline deep sub-micron CMOS processes. Wide band mixer comsumes $9.24 \mathrm{~mW}$ and $9.36 \mathrm{~mW}$ in passive and active case repectively. Reconfigurable mixer is simulated in the frequency range $.5 \mathrm{GHz}$ to $7 \mathrm{GHz}$. II $_{2}$ is $>65$ for both cases. The analytical results shows close to simulted results. With the exception of few very stringent standard, the architechture is suitable for both cases.

\section{REFERENCES}

[1] Hong Zhang; Guican Chen; Xiao Yang, "Fully Differential CMOS LNA and Down-Conversion Mixer for 3-5 GHz MB-OFDM UWB Receivers," Radio-Frequency Integration Technology, 2007. RFIT 007. IEEE International Workshop on , vol., no., pp.54,57, 9-11 Dec. 2007.

[2] Hampel, S.K.; Schmitz, O.; Tiebout, M.; Rolfes, I., "Low-voltage, inductorless folded down-conversion mixer in $65 \mathrm{~nm}$ CMOS for UWB applications," Radio Frequency Integrated Circuits Symposium. RFIC 2009. IEEE, vol., no., pp.119,122, 7-9 June 2009.

[3] Yexin Chen; Na Yan; et al., "Low power, high linearity multi-mode downconversion mixer for SDR," Circuits and Systems (ISCAS), 2013 IEEE International Symposium on, vol., no., pp.737 - 740, may 2013.

[4] Poobuapheun, N.; Wei-Hung Chen; Boos, Z.; Niknejad, A.M., "A 1.5V 0.7-2.5GHz CMOS Quadrature Demodulator for Multi-Band DirectConversion Receivers," Custom Integrated Circuits Conference, 2006. CICC '06. IEEE, vol., no., pp.797,800, 10-13 Sept. 2006.

[5] B Kuan; Fan Xiangning; Li Wei et al., "A wideband current-commutating passive mixer for multi-standard receivers in a 0.18um CMOS," $2013 \mathrm{~J}$. Semicond, Vol. 34, No. 1.

[6] Namsoo Kim; Aparin, V.; Larson, L.E., "A Resistively Degenerated Wideband Passive Mixer With Low Noise Figure and High IIP2 ," Microwave Theory and Techniques, IEEE Transactions on, vol.58, no.4, pp.820,830, April 2010.

[7] Xiaohua Yu; Neihart, N.M., "Analysis and Design of a Reconfigurable Multimode Low-Noise Amplifier Utilizing a Multitap Transformer," Microwave Theory and Techniques, IEEE Transactions on, vol.61, no.3, pp.1236,1246, March 2013.

[8] Geis, A.; Rolain, Y.; Vandersteen, G.; Craninckx, J., "A 0.045mm2 $0.16 \mathrm{GHz}$ reconfigurable multi-band, multi-gain LNA for SDR," Radio Frequency Integrated Circuits Symposium (RFIC), 2010 IEEE , vol., no., pp.123,126, 23-25 May 2010.

[9] Cook, B.W.; Berny, A.D.; Molnar, A.; Lanzisera, S.; Pister, K.S.J., "An Ultra-Low Power 2.4GHz RF Transceiver for Wireless Sensor Networks in $0.13 / \mathrm{spl} \mathrm{mu} / \mathrm{m}$ CMOS with $400 \mathrm{mV}$ Supply and an Integrated Passive RX Front-End," Solid-State Circuits Conference, 2006. ISSCC 2006. Digest of Technical Papers. IEEE International, vol., no., pp.1460,1469, 6-9 Feb. 2006.

[10] Min Wang; Saavedra, C.E., "Reconfigurable broadband mixer with variable conversion gain," Microwave Symposium Digest (MTT), 2011 IEEE MTT-S International, vol., no., pp.1,4, 5-10 June 2011.

[11] Jiangtao Xu; Saavedra, C.E.; Guican Chen, "A 12 GHz-Bandwidth CMOS Mixer With Variable Conversion Gain Capability," Microwave and Wireless Components Letters, IEEE Volume: 21, Issue: 10 Publication Year: 2011, Page(s): 565 - 567.

[12] Kuan Ba; Xiangning Fan et al.,"A CMOS Reconfigurable Passive Mixer with Digitally-Controllable Gain," Radio-Frequency Integration Technology (RFIT), 2014 IEEE International Symposium, Page(s): 1-3.

[13] R. Circa; D. Pienkowski; G. Bck; and R. Wittmann, "Reconfigurable UMTS/WLAN RF receiver, in Proc. 3rd Software Radios Workshop, Karlsruhe, Germany, Mar. 2004, pp. 6571.

[14] Kakerow, R.; Mueller, M.; Pienkowski, D.; Circa, R.; Boeck, G., "Reconfigurable receiver approach for $4 \mathrm{G}$ terminals and beyond," IEEENEWCAS Conference, 2005. The 3rd International, vol., no., pp.9,12, 19-22 June 2005 